\title{
Advances in Oncology in US and Japan: Focusing on Cancer and Infectious Diseases
}

\author{
Hiroto Nakano $^{\mathrm{a}}$, Takuto Miyamoto ${ }^{\mathrm{b}, \mathrm{c}}$, Yelena Y. Janjigian ${ }^{\mathrm{d}}$, Sohtaro Mine ${ }^{\mathrm{e}}$, Hiroaki Mitsuya ${ }^{\mathrm{f}, \mathrm{g}, \mathrm{h}}$, \\ Naoto T. Ueno ${ }^{\mathrm{i}}$, Elad Sharon', Shunsuke Sakaik, William C. Timmer ${ }^{\mathrm{l}}$, Shuichiro Nakabo ${ }^{\mathrm{m}}$, \\ Tomoko Ikeuchi $^{n}$, Saori Fujiwara ${ }^{o}$, Minori Kinjo ${ }^{p}$, Tadashi Inuzuka ${ }^{q}$, Hayato Kume ${ }^{r}$, Keisuke Shirai ${ }^{\mathrm{s}}$, \\ Norihiro Yamaguchit, Kazuaki Takabe ${ }^{\mathrm{u}, \mathrm{v}, \mathrm{w}, \mathrm{x}, \mathrm{y}, \mathrm{z}}$, Naoko Takebe ${ }^{\# *}$
}

\begin{abstract}
This is a review article based on the international symposium report of the "US-Japan Conference on Advances in Oncology: Cancer and Infectious Diseases" held online on June 25, 2021, which provided an update on the association between oncology and infectious disease research from cutting-edge basic science to high-impact clinical trials.
\end{abstract}

Keywords: Oncology; Infectious diseases; Oncogenesis; Translational research; Immunotherapy; Clinical trials; Career development; CoRe Partnership

\section{Introduction}

Since 2010, the Embassy of Japan in the United States of America and recently the Japan Agency for Medical Research and Development (AMED) Washington, D.C. Office have organ-

Manuscript submitted October 5, 2021, accepted December 2, 2021

Published online December 13, 2021

${ }^{a}$ Translational Genetics and Genomics Unit, National Institute of Arthritis and Musculoskeletal and Skin Diseases (NIAMS), National Institutes of Health (NIH), Bethesda, MD 20892, USA

${ }^{b}$ Washington, D.C. Office, Japan Agency for Medical Research and Development (AMED), District of Columbia, USA

${ }^{\mathrm{c}}$ Ministry of Education, Culture, Sports, Science and Technology, Tokyo, Japan

${ }^{\mathrm{d}}$ Gastrointestinal Oncology Service, Department of Medicine, Memorial Sloan Kettering Cancer Center, New York, NY 10065, USA

${ }^{e}$ Medical Virology Section, Laboratory of Infectious Diseases, National Institutes of Health, Bethesda, MD 20892, USA

${ }^{f}$ Experimental Retrovirology Section, HIV and AIDS Malignancy Branch, National Cancer Institute (NCI), National Institutes of Health (NIH), Bethesda, MD 20892, USA

gDepartment of Refractory Viral Infections, National Center for Global Health and Medicine Research Institute, Tokyo, Japan

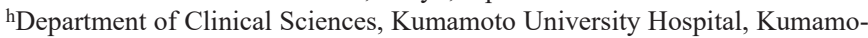
to, Japan

iDepartment of Breast Medical Oncology, The University of Texas MD Anderson Cancer Center, Houston, TX, USA

(To be continued on page 192) ized a series of workshops on clinical trials in oncology with Kazuaki Takabe and Naoko Takebe as course directors, primarily targeting Japanese medical researchers in Washington, DC, Maryland, and Virginia area (Table 1). The workshops were designed to share the knowledge of accomplished researchers on up-to-date topics given the importance of bridging basic research and clinical trials and to help participants develop their careers not only in the US but also in Japan after their return to their home country. It aimed to develop next-generation medical investigators from international perspectives.

Following the success of the seventh workshop in 2019, volunteers led by Japanese medical researchers formed the steering committee to hold the eighth workshop in 2020. However, the workshop was put on hold due to the impact of the coronavirus disease 2019 (COVID-19) pandemic. Meanwhile, in April 2020, a summit-level meeting was held between the US and Japan, and the US-Japan Competitiveness and Resilience (CoRe) Partnership was agreed. The partnership intends further strengthen mutual research activities between the US and Japan. For such backgrounds, in 2021, the steering committee decided to proceed to a renewed online conference on oncology.

The steering committee identified two objectives of the conference: 1) to strengthen research collaboration between both countries through distinguished lectures and interactive discussions on cross-cutting research areas of immunology, infectious diseases, and cancer, which are of increasing interest due to the pandemic; and 2) to enable young researchers to consider enhancing their career through lectures and discussions on a wide range of topics, including not only the latest research information, but also career development, securing research funding, and building research schemes.

The conference was held online on June 25, 2021, co-hosted by the Embassy of Japan and AMED Washington, D.C. Office. Washington CORE LLC, independent research and consulting firm also joined as supporting members. The conference was announced via a Facebook page (https://www.facebook.com/USJP. ONCOLOGY/), the Embassy and AMED websites, and e-newsletters. An online registration site was provided. Over 100 participants attended the conference from the United States and Japan despite time-zone difference. The participants were from diverse institutions, universities, private companies, the US government agencies including the National Institutes of Health (NIH), Food and Drug Administration (FDA), National Center for Global 
Table 1. History of the US-Japan Workshops

\begin{tabular}{|c|c|c|}
\hline Date & Titles/themes & Venue \\
\hline June 12,2010 & $\begin{array}{l}\text { The US-Japan Clinical Trials in Oncology Workshop } \\
\text { Career Development in Clinical Oncology }\end{array}$ & The old residence of the Japanese ambassador \\
\hline April 17, 2015 & The 3rd US-Japan Clinical Trials in Oncology Workshop & The old residence of the Japanese ambassador \\
\hline June 9, 2016 & $\begin{array}{l}\text { The 4th US-Japan Clinical Trials in Oncology Workshop } \\
\text { (Focusing on precision medicine) }\end{array}$ & Japan Information \& Culture Center (JICC) \\
\hline June 6,2018 & $\begin{array}{l}\text { The 6th US-Japan Clinical Trials in Oncology Workshop } \\
\text { Emergence of AI in Medicine, Good Friend or Potential Enemy }\end{array}$ & The old residence of the Japanese ambassador \\
\hline June 5, 2019 & $\begin{array}{l}\text { The 7th US-Japan Clinical Trials in Oncology Workshop } \\
\text { Develop Your Career }\end{array}$ & The old residence of the Japanese ambassador \\
\hline
\end{tabular}

Health and Medicine in Tokyo, Japanese government agencies including the Cabinet Office, the Ministry of Health, Labour and Welfare (MHLW), the Ministry of Education, Culture, Sports, Science and Technology (MEXT) of Japan, and AMED.

\section{Opening Remarks}

The event started with Ambassador Tamaki Tsukada, Minister Plenipotentiary, and the Deputy Chief of Mission at the Embassy of Japan in the United States.

He noted the importance of the theme of this conference, "Cancer and Infectious Diseases", which is at the intersection of cancer, infectious diseases, and immunology through the past year and a half of COVID-19 we experienced. The pandemic raises fundamental questions about its origins and mechanisms, and that the science and policy communities will need to answer these questions for years to come.

He also mentioned the assets of our predecessors for the US-Japan research cooperation, including that Prime Minister Sato and President Johnson launched the US-Japan Cooperative Medical Sciences Program (USJCMSP) in 1965. The USJCMSP, which continues to this day, established the foundation of research exchange and cooperation and brought about the great sea of activities we see today between Japan and the United States.

In April 2021, Prime Minister Suga and President Biden agreed to the US-Japan CoRe Partnership, which includes continued US-Japan collaboration in cancer research. He noted this conference is one of the small but significant activities under this agreement.

In conclusion, he expressed his expectations for the excellent and stimulating discussions at the conference.

\section{Success in Academia: A Journey not a Destina- tion}

Yelena Janjigian, M.D., is a board-certified medical oncologist who specializes in the treatment of cancers of the gastrointes- tinal tract. Her clinical practice and research are focused on improving treatment outcomes for people with esophageal and stomach cancers. As Chief of the Gastrointestinal Oncology Service at Memorial Sloan Kettering Cancer Center (MSK$\mathrm{CC}$ ), her mission is to foster research and innovation that transform the future of GI oncology to help patients worldwide. Her research has been supported by grants from the National Cancer Institute (NCI), the American Society of Clinical Oncology (ASCO), and The Society of MSKCC.

She came as a refugee to the United States in 1991. She is of Armenian descent and learned English when she was 14. She received her medical degree from NYU School of Medicine in 2003 and has been in practice for over 20 years.

The presentation began by discussing her journey as a medical oncologist, starting as a lung cancer doctor at MSK$\mathrm{CC}$, focusing on acquired resistance. She eventually pivoted to become a gastrointestinal oncologist given the major burden of gastric cancer, becoming the Chief of Gastrointestinal Oncology in 2018. This unit currently has 102 active therapeutic clinical trials.

One of the current therapeutic targets of MSKCC is immunotherapy in esophagogastric adenocarcinoma; a drug for this, nivolumab (programmed cell death protein 1 (PD-1) blocker), is currently approved in Asia, while pembrolizumab (PD-1 receptor blocker) is approved in > 3rd line in the US MSKCC is working with Bristol Myers Squibb (BMS) on a clinical trial of this drug in the United States to create a therapeutic option to address gastric cancer early on before other complications develop.

Next, Yelena Janjigian discussed the CheckMate 649 Study Design, a randomized, open-label phase 3 study. Eligibility criteria for the study include previously untreated, unresectable, advanced, or metastatic gastric/gastroesophageal junction (GEJ)/esophageal adenocarcinoma; no known human epidermal growth factor receptor 2 (HER2)-positive status, and Eastern Cooperative Oncology Group (ECOG) Performance Status (PS) 0-1.

This study led to the FDA approval of nivolumab plus chemotherapy as a treatment for gastric cancer based on improved survival rates, which was one of the first FDA approv- 
als in this field in over a decade.

She then discussed a case of Microsatellite Instability High (MSI-H) stage IV gastric cancer involving circulating tumor DNA (ctDNA) monitoring. She presented the example of a woman with profound symptoms, with biomarkers showing the patient was HER2-positive, programmed death-ligand 1 (PD-L1)-positive, indicating a high mutational burden. This type of cancer is typically resistant to chemotherapy, with immunotherapy presenting a more attractive option (pembrolizumab). The approach for early-stage MSI-H tumors is to use ctDNA as an indicator of whether immunotherapy should be used instead of chemotherapy.

For the last few minutes, Yelena Janjigian discussed HER2, an important biomarker for gastric cancer. HER2 receptor inhibitor trastuzumab has been an important treatment for this disease for at least 10 years, but beyond this, it has been difficult to move forward in the field of gastric cancer treatment. A lot of the other drugs commonly used in breast cancer have not been shown to be effective for gastric cancer. At the genomic level, there may be clues as to intrinsic resistance to trastuzumab by showing the correlation between these genomic factors and responsiveness to the therapy. In gastric cancers, there is a lot of variability in the level of HER2, which could explain varying response rates to treatment. Furthermore, as a consequence of delayed immunotherapy treatment with subsequent efforts to improve on the HER2-directed therapy in later lines of therapy, patients can lose HER2 and thus not respond as well to HER2-directed therapy [1]. For HER2-negative diseases, a combination of tyrosine kinase inhibitors (TKIs) plus immunotherapy may help overcome resistance.

A key lesson in the HER2-positive gastric cancer is that targeting disease early and targeting it in a different way (other than a combination of different TKIs) may be the better way to go. MSK designed a phase 2 trial with dual PD-1 and anti-HER2 blockade with an overall response rate of $91 \%$ with a $100 \%$ disease control rate. Five to six years later, some of the patients were even cured (https://ascopubs.org/doi/ abs/10.1200/JCO.2021.39.15_suppl.4013).

Based on these results, MKSCC launched the Keynote 811 study, which showed similar response rates as the phase 2 study using pembrolizumab. The eligibility criteria of this study included unresectable or metastatic gastric or GEJ adenocarcinoma; no prior systemic therapy in an advanced setting; HER2-positive tumor by central review and ECOG PS 0 or 1 . The study involved two groups, one of which received pembrolizumab and trastuzumab, while the other received a placebo and trastuzumab, showing significant benefits from the combination of pembrolizumab and trastuzumab. Both Keynote 811 and Checkmate 649 led to FDA approvals [2].

Other work is occurring in preclinical models in the Ganesh lab to understand mechanisms of the synergy of these combinations and how to target other subsets, using tools such as a gastric cancer organoid biobank. There is a very busy gastric cancer practice at MSKCC that allows the collection of these samples.

For adenocarcinoma, MSKCC is focusing research on gastric cancer, including adenocarcinoma with its recent FDA approvals such as: 1) nivolumab with folinic acid, fluoroura- cil, and oxaliplatin combination therapy (FOLFOX) approved in the US in first-line metastatic gastric cancer irrespective of PD-L1 status, and 2) pembrolizumab trastuzumab and chemotherapy approved in the US in HER2-positive disease.

Finally, she also discussed a study at MSKCC related to COVID-19 that examined the association of severe cases with types of cancer to determine how therapies might increase the risk of hospitalization, finding that severe illness was very rare in association with gastric cancer. The study concluded that chemotherapy was not an independent prognostic factor for severe COVID-19 nor was immunotherapy; therefore cancer treatment could continue safely for GI cancers during the pandemic.

\section{Viral Infections and Carcinogenesis - Surpris- ingly Large Public Health Burden}

Sohtaro Mine, M.D., Pharm.D., Ph.D., works as a postdoctoral fellow at a US institute studying clinical aspects of viral infections and associated tumors. He has authored papers specializing in lymphoma, hematological malignancies, Epstein-Barr virus (EBV) and human herpesvirus $8(\mathrm{HHV}-8)$. Before working in the USA, he worked at the National Center for Global Health and Medicine Hospital and the National Institute of Infectious Diseases in Japan as a pathologist and researcher in pathological diagnosis. His research involved diseases caused by human herpesviruses such as the EBV [3].

The presentation started by discussing the connection between infections and carcinogenesis, which is a major issue among the research community, but less of a public concern despite a large public health burden stemming from this issue.

Around $30 \%$ of cancer is caused by infectious diseases. In 1911, Peyton Rous discovered a sarcoma in chicken that could be transmitted to another chicken by a virus [4], constituting the first discovery of an oncogenic virus. The first oncogenic virus in humans was not discovered until 1964 with the breakthrough demonstrating a connection between EBV, and Burkitt lymphoma [5]. Further research demonstrated other connections, such as the association between the human papillomavirus (HPV) and cervical cancer, among others.

These issues are not as distinct of a public health burden in developed countries but constitute significant challenges in developing countries that struggle with cancers stemming from HPV, hepatitis B and C, Helicobacter pylori (H. pylori), and HHV-8. Types of cancers from these infectious diseases are shown in Table 2.

Regarding HPV, there are over 150 - 200 different strains with certain high-risk types known to cause various types of cancer. These pose a challenge in developing countries due to the lack of access to vaccines. The carcinogenesis of HPV involves factors in its genome that have a carcinogenic effect in cells (oncogene E6 and E7 are expressed in cells, with E6 preventing the function of tumor protein p53 (TP53) and E7 interacting with the retinoblastoma protein $(\mathrm{pRb})$-associated pocket proteins which are negative cell-cycle regulators resulting in the enhanced phosphorylation and degradation of the protein, thus halting apoptosis and leading to propagation of 
Table 2. Cancer Caused by Infectious Diseases

\begin{tabular}{ll}
\hline Pathogens & Tumor \\
\hline Helicobacter pylori & Gastric carcinoma, mucosa-associated lymphoid tissue (MALT) lymphoma \\
Epstein-Barr virus (EBV) & Nasopharyngeal cancer, lymphoma, gastric cancer, etc. \\
$\begin{array}{l}\text { Human papilloma virus (HPV) } \\
\text { Hepatitis B virus (HBV), hepatitis C virus }\end{array}$ & Cervical cancer, anal cancer, vulvar cancer, vaginal cancer, and cancer of the mid-pharynx \\
(HCV) & Hepatocellular carcinoma \\
Human herpes virus 8 (HHV-8, KSHV) & Kaposi's sarcoma, primary effusion lymphoma, Castleman's disease \\
Merkel cell polyomavirus (MCPyV) & Merkel cell carcinoma \\
Human T-cell leukemia virus type I (HTLV-1) & Adult T-cell leukemia/lymphoma (ATLL) \\
Clonorchis sinensis & Bile duct cell carcinoma \\
Schistosoma Haematobium & Bladder carcinoma \\
\hline
\end{tabular}

uncontrolled cell growth).

Hepatitis B is a DNA virus, while hepatitis C is an RNA virus. The vaccine is the only option for hepatitis $\mathrm{B}$ virus, while curative treatment options have become available for hepatitis C. Both diseases cause inflammation that leads to carcinogenesis in tissues and organs.

Human T-cell leukemia is an adult T-cell leukemia common among patients from Kyushu, Japan. The retrovirus named human T-cell leukemia virus (HTLV-1) was discovered that causes this cancer, in which cancerous cells proliferate indefinitely from infected $\mathrm{T}$ cells. This virus can be transmitted vertically in the form of breast milk, or horizontally via blood transfusion, or sexually transmitted manner. There is no vaccine for this virus, but research has shown that transmission can be prevented through public education and screening carriers.

Merkel cell carcinoma polyomavirus (MCPyV) is an infection that causes cancer to arise from Merkel cells, which are in the skin in very close proximity to nerve endings, also known as skin neuroendocrine carcinoma. It is the second leading cause of death from skin cancer, after melanoma, despite its rarity.

Next, he discussed the carcinogenesis of HHV-8 (KSHV); there are many factors encoded in the virus that can be linked to cancers that signal for cells to proliferate, but it is not clear what combination of factors leads to cancers. For EBV, which can lead to a wide range of cancers (nasopharyngeal carcinoma, autoimmune diseases, and other malignant tumors), the story is similar in that there is no single factor that has been identified as a deciding factor for causing cancer.

Finally, he discussed the issue of acquired immunodeficiency syndrome (AIDS)-related lymphoma in the context of the issue in which human immunodeficiency virus (HIV)-infected individuals experience more than 10 times higher rates of malignant lymphoma than healthy individuals. Based on an analysis of 225 cases in Japan with a mean age of 44 years old, many herpesvirus-related malignancies were discovered, along with EBV-associated lymphoma [6]. This research demonstrates the critical role of the patient's immune status in the incidence of cancer; further research is looking into what immune cells are important for preventing these cancers.
Sohtaro Mine's conclusions included that there is a surprising number of cancer-causing infectious diseases and that the most attractive option is the prevention of the infection; HPV and HBV infection can be prevented by a vaccine, while hepatitis $\mathrm{C}$ virus $(\mathrm{HCV})$ can be treated with medicine; H. pylori can be eradicated using antibiotics and; immune mechanisms and carcinogenesis are closely related.

\section{Battle Against the Killer Viruses: HIV and Se- vere Acute Respiratory Syndrome Coronavirus 2 (SARS-CoV-2)}

\section{Development of anti-SARS-CoV-2 therapeutics and COVID-19-convalescent plasma transfer therapy}

Hiroaki Mitsuya, M.D., Ph.D., is a Japanese virologist and is Chief of Experimental Retrovirology Section of the NCI, NIH, Bethesda, Maryland, USA, former Chairman and Professor of Department of Hematology and Rheumatology, and Director of Division of Infectious Diseases at the National Kumamoto University School of Medicine, Kumamoto, Japan. He has also assumed the position of Director-General in the National Center for Global Health and Medicine Research Institute, Tokyo, Japan since April 2016.

Hiroaki Mitsuya obtained his M.D. and Ph.D. from Kumamoto University School of Medicine in Japan. After receiving immunology/hematology/oncology training at Kumamoto University Hospital, he joined the NCI, NIH, in 1982. In 1985, he discovered the first three therapeutics for the treatment of AIDS and guided much of their preclinical development. In 2006, he was awarded the first NIH World AIDS Day Award for his pioneering work in developing the treatment of HIV infection and AIDS (https://academicmedicaleducation.com/ hiroaki-mitsuya-md-phd).

The beginning of his presentation centered around the challenges in AIDS treatment in the middle of the 1980s and 1990 s, such as insufficient potency of early time AIDS therapeutics, drug-related toxicities, and the emergence of drugresistant HIV variants. The HIV/AIDS science, however, has 


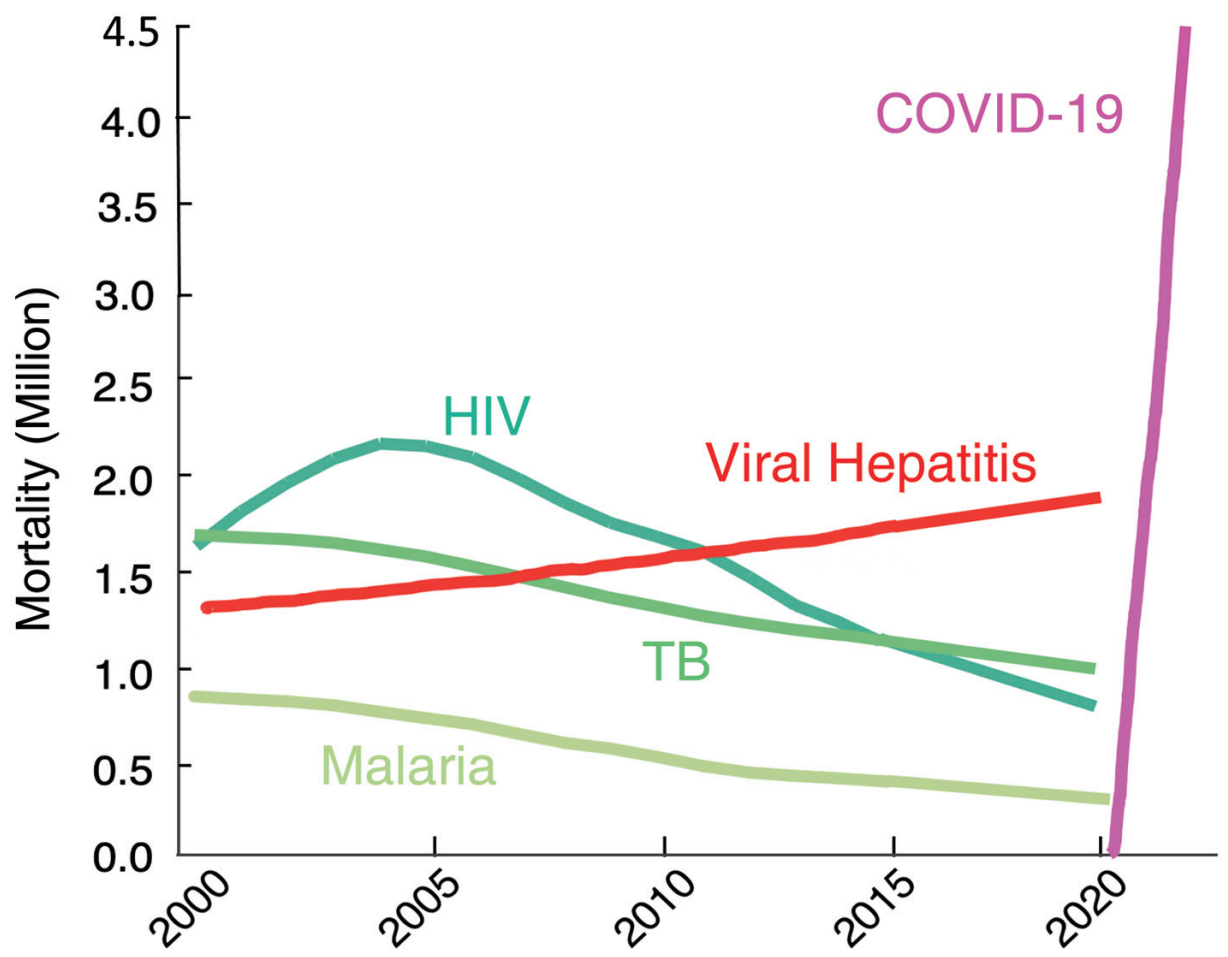

Figure 1. The death toll from coronavirus disease 2019 (COVID-19) goes far beyond that of any of human immunodeficiency virus (HIV)/tuberculosis (TB)/malaria. The number of deaths from COVID-19 surpassed by far that of fatalities of the top three communicable diseases (CDs): HIV/acquired immunodeficiency syndrome (AIDS), tuberculosis, and malaria. https://www.worldlifeexpectancy.com/world-rankings-total-deaths; https://www.nytimes.com/interactive/2021/world/covid-vaccinations-tracker.html.

solved many such problems and HIV/AIDS has now become a controllable chronic infection and the life expectancy of persons with HIV/AIDS has become the same or very close to that of persons without HIV/AIDS. Yet, we are facing new challenges different from those we had at the beginning of AIDS therapy. Even now, we can achieve only partial restoration of immunologic functions once lost, and persons with HIV/AIDS develop various cancers as a consequence of survival prolongation and suffer from AIDS-related central nervous system (CNS) abnormalities. The increased cost of antiviral therapy is also a world-wide ethical challenge. To solve such problems at least in part, we have various new drugs in the pipeline. They include new agents of different classes, novel mechanisms, and different formats including long-acting oral products (weekly/ monthly) as well as annual implants. Recent breakthroughs include the development of pre-exposure prophylaxis (PrEP) implant using islatravir (EFdA, nucleoside reverse transcriptase translocation inhibitor), which Mitsuya and his group have recently invented (https://www.poz.com/article/islatravir-showspromise-oncemonthly-oral-prep). The islatravir PrEP implant can be placed under the skin, providing protection against HIV infection over a year without significant adverse effects. The novel drug islatravir can be a "game-changer" and "paradigm shift" in the treatment and prophylaxis of HIV/AIDS (https:// www.poz.com/article/islatravir-shows-promise-oncemonthlyoral-prep, https://www.biospace.com/article/hiv-drugmakersgilead-and-merck-partner-to-develop-new-hiv-treatment).

The second portion of his presentation discussed the ur- gent global health crisis, SARS-CoV-2-caused COVID-19. The continuing spread of the infection of SARS-CoV-2 has been a global and grave public health threat. The number of casualties from COVID-19 has surpassed by far that of fatalities from the top three communicable diseases: HIV/AIDS, tuberculosis, and malaria (Fig. 1) [7]. The toll of hepatitis still continues to rise; however, the toll from COVID-19 has surpassed immediately after the appearance of SARS-CoV-2/ COVID-19. These numbers reveal how grim the emergence of a new hard-to-treat and highly contagious infectious pathogen can be to mankind. As for the strategy in the fight with SARSCoV-2/COVID-19, vaccination is one of the most effective prophylactic health measures. In fact, mRNA-based vaccines have proven to be exceedingly promising key strategy for curbing the current COVID-19 pandemic. Yet, a number of people have not been vaccinated as of the time of the current meeting and no SARS-CoV-2-specific effective therapeutics are available except remdesivir, a SARS-CoV-2's RNA-dependent RNA polymerase. Thus, more immediate way of treatment is required. In that regard, plasmas from patients convalescent from viral infections could serve as an immediately available treatment option (Fig. 2) [8]. That treatment modality involves infusions of infectious-pathogen-specific antibodies present in human convalescent plasma to bring about improvement in the disease condition. However, the efficacy of plasma infusion therapy has been controversial, mainly because most clinical trials to-date have not been controlled or randomized, despite the studies showing that fatality among those receiving plas- 


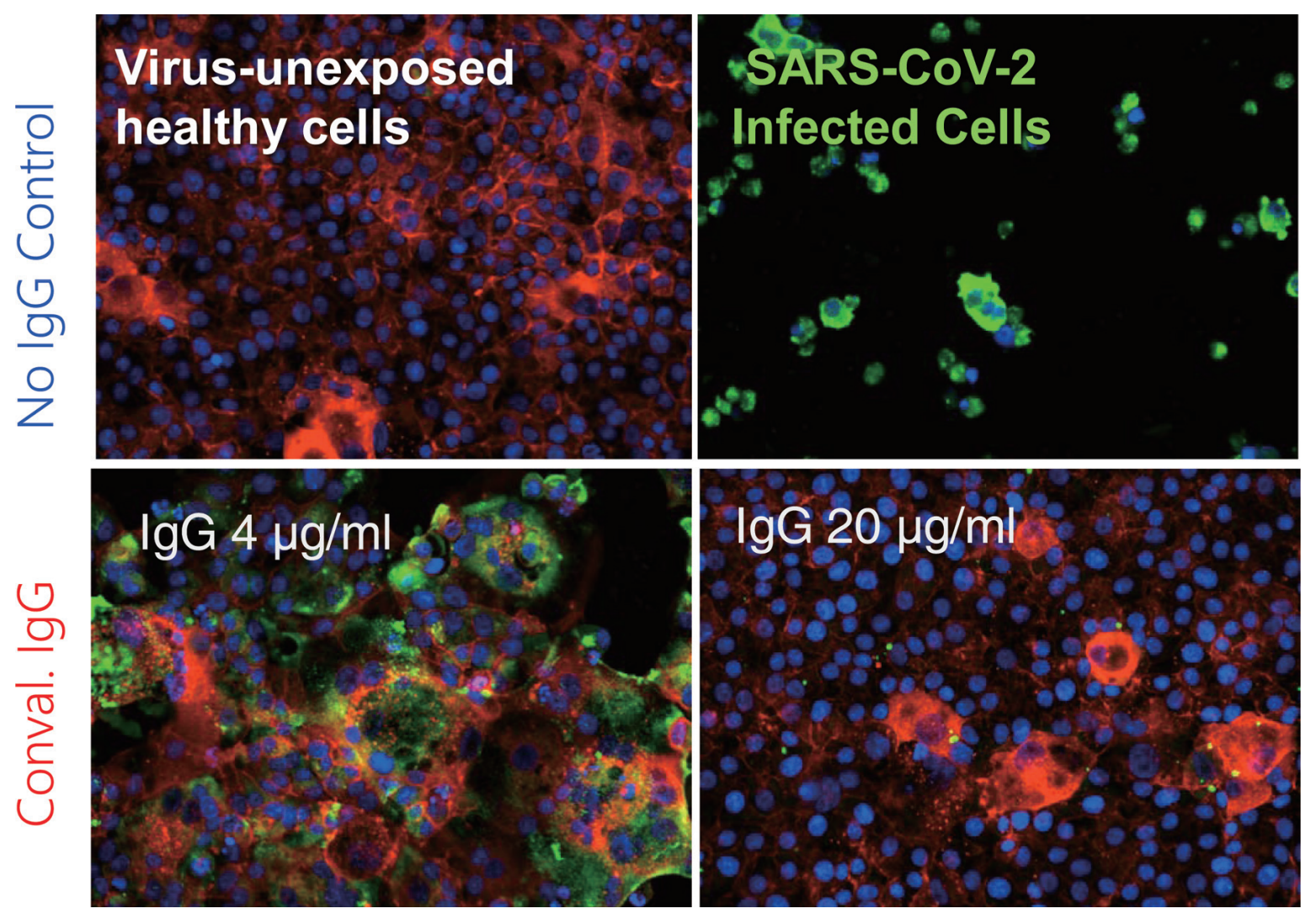

Figure 2. Coronavirus disease 2019 (COVID-19)-convalescent plasma-derived IgG completely blocks the infectivity and cytopathic effect of severe acute respiratory syndrome coronavirus 2 (SARS-CoV-2) in test tube. The method, immunocytochemistry, reveals robust cytoskeleton (filamentous actins stained in red) in virus-unexposed healthy target VeroE6 ${ }^{\text {TMPRSS2}}$ cells (upper left). If infected with SARS-CoV-2, cytoskeletons are destroyed, and viral antigens are produced and stain in green and the cells are eventually destroyed (upper right). However, if the cells are exposed to SARS-CoV-2 and cultured in the presence of $4 \mu \mathrm{g} / \mathrm{mL}$ IgG purified from a serum sample from a COVID-19-convalescent patient, they are partially protected from the infection. In the presence of IgG $20 \mu \mathrm{g} / \mathrm{mL}$, the infectivity and cytopathic effect of the virus is completely blocked. These data strongly suggest that a sufficient amount of SARS-CoV-2-neutralizing antibody can completely protect target cells from SARS-CoV-2. Cellular actin filaments, cellular nuclei, and viral antigens are stained in red (TexasRed-X), blue (DAPI), and green (Alexa Fluor 488), respectively [8].

ma was lower in certain viral diseases. Mitsuya and his group have demonstrated that, in SARS-CoV-2-infected hamster model, COVID-19-convalescent plasmas significantly reduce SARS-CoV-2 titers in the lung of Syrian hamsters and prevent pneumonia from occurring (Fig. 3) [9]. Mitsuya and his group have started a randomized clinical trial to evaluate the effect of highly neutralizing COVID-19-convalescent plasma transfer to seniors and those at high risks of having severe COVID-19, which began in February 2021 in multiple medical centers in Tokyo and is ongoing.

Hiroaki Mitsuya also stressed that the development of SARS-CoV-2-specific therapeutics is essential to curb the pandemic. In terms of COVID-19 management, remdesivir, dexamethasone, baricitinib, and interleukin-6 (IL-6) pathway inhibitors are the only recommended agents at the time of meeting for severely ill patients with COVID-19, although the efficacy of such agents is very limited and COVID-specific therapeutics are still in the middle of development.

From the perspective of drug development, it is noteworthy that the algorithm of HIV/AIDS drug development appears to serve as the basis of the study of potential COVID-19 drugs. In fact, the structure of HIV protease and its inhibitors should serve as templates for the development of SARS-CoV-2 protease inhibitors [10]. In this regard, Mitsuya and his group have identified a group of small-molecule compounds highly active against SARS-CoV-2 protease (main protease; Mro). Figure 4 illustrates that a SARS-CoV-2-M ${ }^{\text {pro }}$-specific inhibitor, GRL-2420/5h, tightly binds to the active site of SARS-CoV-

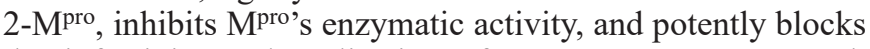
the infectivity and replication of SARS-CoV-2 [11]. Such compounds should serve as promising leads for developing more potent and safer compounds that block the infectivity of SARS-CoV-2. In fact, he has designed, synthesized, and identified multiple highly potent and non-cytotoxic small-molecule compounds. Such compounds are now under development toward clinical application.

His presentation closed with the discussion of his recent findings that after the structure-based re-design and synthesis, multiple far-more potent $\mathrm{M}^{\text {pro }}$ inhibitors have been identified by his group. 
Healthy Plasma
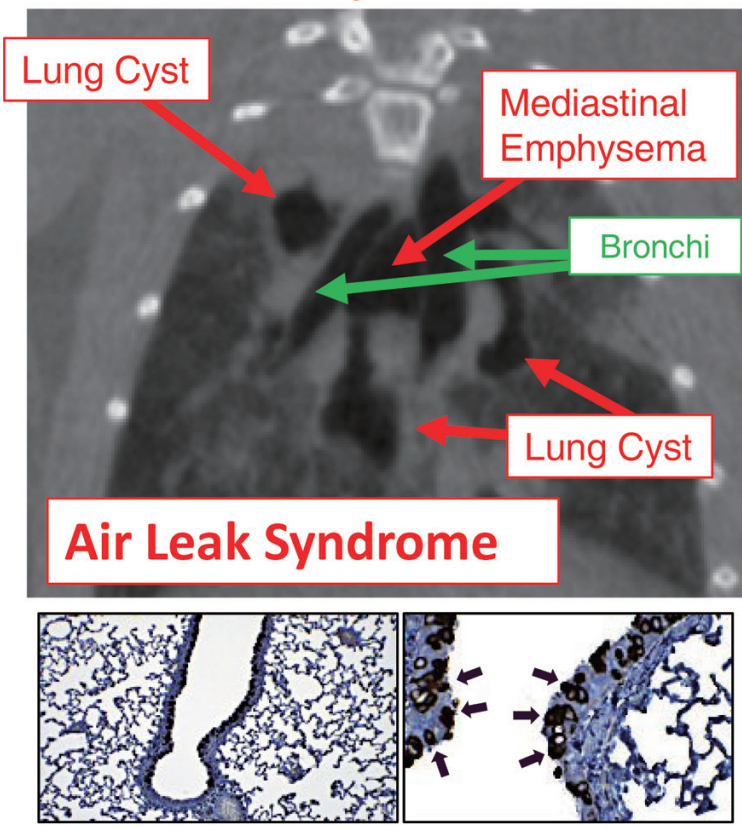

Conval. PlasmaD43
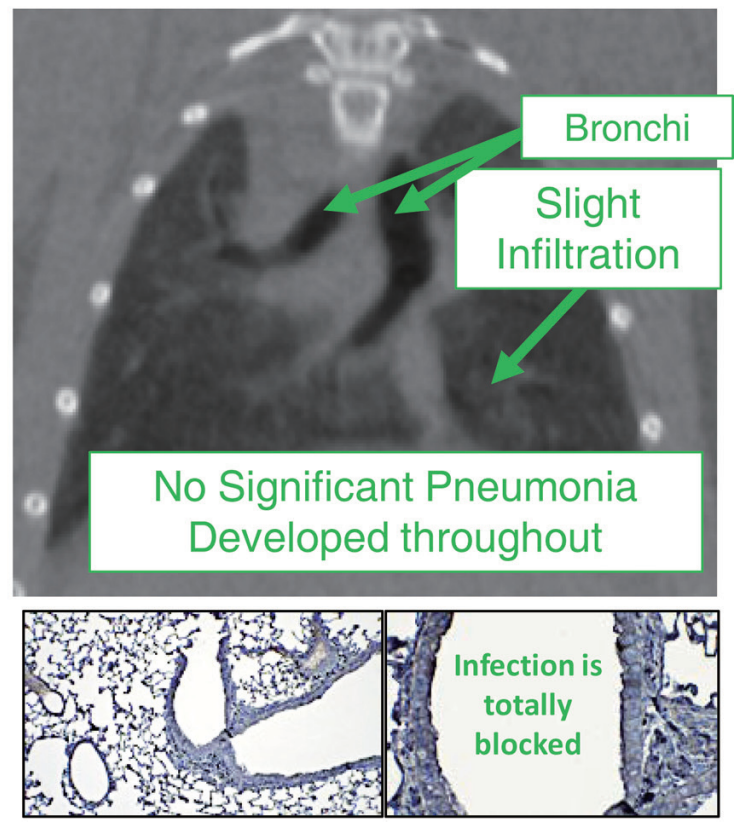

Figure 3. Hamsters receiving coronavirus disease 2019 (COVID-19)-convalescent plasma are protected from having pneumonia. Micro-computed tomography $(\mathrm{mCT})$ scan was used to image the hamster lungs. For immunohistochemistry, the IgG fraction of serum from a convalescent COVID-19 individual was employed as the primary antibody and peroxidase conjugated goat polyclonal anti-human IgG antibody was used as the secondary antibody. For visualization, 3,3'-diaminobenzidine (DAB)peroxidase enzyme reaction was performed. While Syrian hamsters, which were SARS-CoV-2-inoculated and received control healthy plasma developed severe pneumonia by day 8 following viral inoculation (upper left), hamsters which were inoculated with severe acute respiratory syndrome coronavirus 2 (SARS-CoV-2) but received COVID-19-convalescent human plasma (D43) were protected from infection by SARS-CoV-2 and developed no pneumonia (upper right). The two lower left insets show that many airway cells of the hamster had been infected with SARS-CoV-2 and stained in dark brown; however, the two lower right insets show no infection on the airway cells [9].

\section{Special message}

Naoto Ueno, M.D., Ph.D., is a Professor of Medicine at The University of Texas MD Anderson Cancer Center. His research is in translational breast cancer research in cancer biology and molecular therapeutics, with expertise in the key research areas of epidermal growth factor receptor (EGFR)/extracellular signal-regulated kinases (ERK)/c-Jun N-terminal kinase (JNK) signaling and in vivo modeling. He is best known for his preclinical development of targeted and immunotherapy in breast cancer. Further, he conducts many new phase I/II studies based on his translational discovery. In addition, he is the Executive Director of the Morgan Welch Inflammatory Breast Cancer Program and Clinic and Section Chief of the Translational Breast Cancer Research at the Department of Breast Medical Oncology.

He presented the concept and activities of CancerX, a cancer advocacy group in Japan that aims to create a society that addresses impending doom/death and fear/anxiety associated with cancer diagnoses and treatment. The genuinely impactful research outcome stems from designing a society that can support patients with cancer and their caregiver from diverse perspectives. Collaboration among various fields can reduce unconscious bias and provide support to cancer patients with more well-rounded support, with the goal that a cancer diagnosis invokes a "So what?" response from the patient. The group uses communication and outreach to empower patients and caregivers to work towards this goal.

CancerX has worked with Yokohama City to develop a cancer preparation strategy. It also hosted the World Cancer Summit three times since 2019 attended by prominent stakeholders in the cancer research area and non-cancer-related CEOs, and a Chief Cabinet Secretary of the Japanese government.

For example, given that chemotherapy suppresses the immune system, the medical community has been developing new antibiotics and diagnostic tools. However, from a broader societal perspective, there is an inadequacy to support the immunocompromised patients to be integrated into society. For example, those undergoing chemotherapy, including Naoto Ueno himself, are advised to avoid certain restaurants (e.g., sushi) to avoid risks to their compromised immune systems at one point in their life. How can we provide an opportunity for immunocompromised patients to enjoy eating outside despite their health status? More matrix solutions are needed, not just developing a treatment for immunocompromised related to infection. He called for a better strategy for the cancer community to engage with the rest of the world and raise awareness about these issues and the need for providing a solution; he 


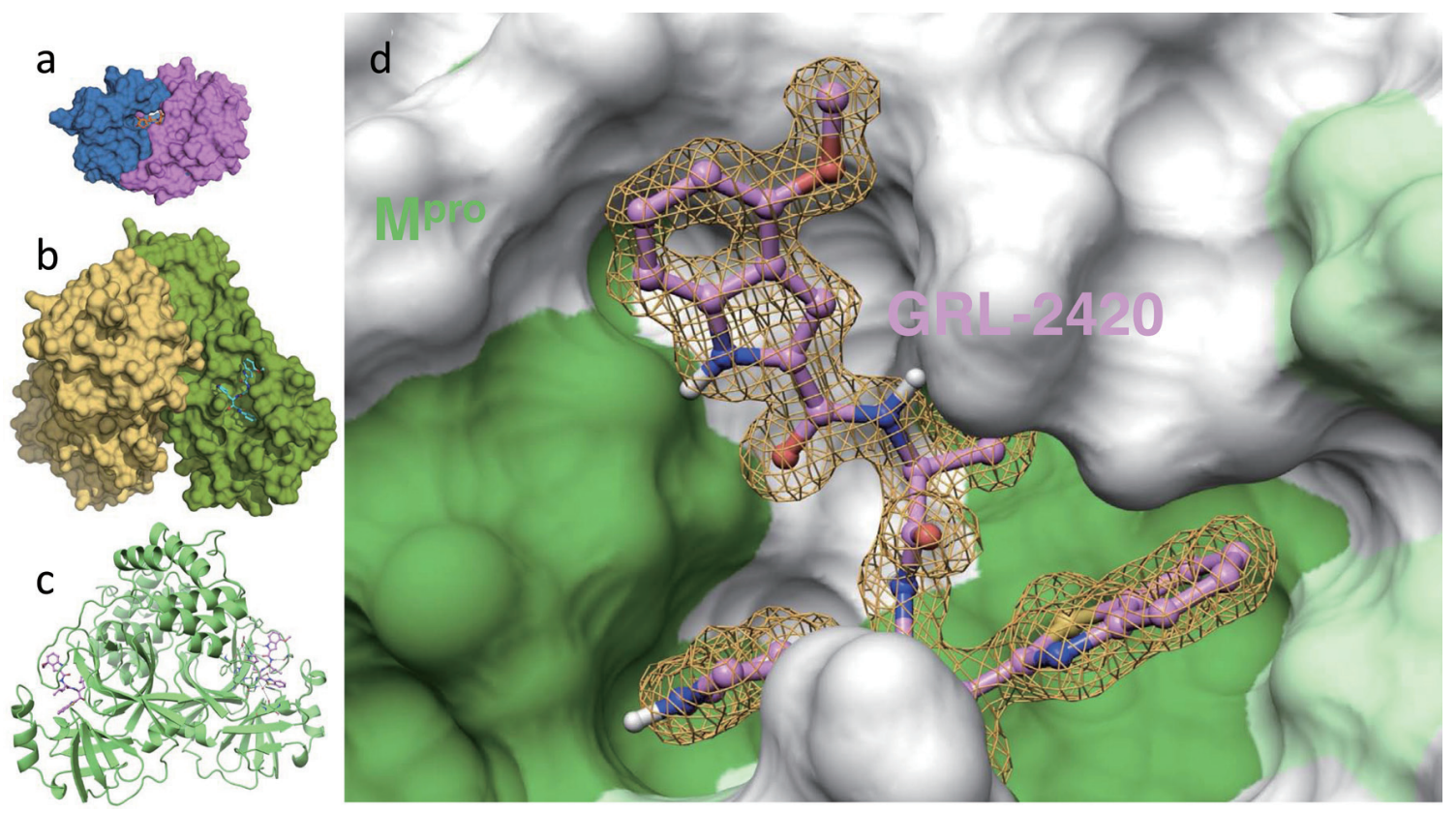

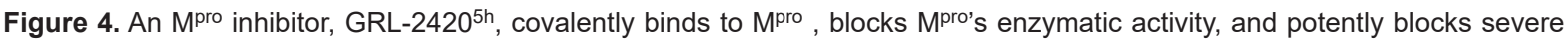
acute respiratory syndrome coronavirus 2 (SARS-CoV-2) infectivity and replication. Inset (a) shows human immunodeficiency virus (HIV)'s protease bound by its inhibitor, darunavir, and insets (b) and (c) show SARS-CoV-2's protease (Mpro) bound by an Mpro inhibitor, GRL-2420/5h, in the forms of surface and ribbon representations, respectively. Note that the size of $\mathrm{M}^{\text {pro }}$ is three-times larger than that of HIV's protease. Inset d shows the hydrophobic cavity of Mpro's enzymatic active site, into which GRL-2420/5h snugly lodges and blocks the enzymatic activity. It is of note that the algorithm of HIV/acquired immunodeficiency syndrome (AIDS) drug development serves as the basis of study of potential COVID-19 drugs [11].

called for the outreach of researchers and healthcare providers to communities that have "nothing to do with cancer" to create a society where living with cancer is far easier and less frightening.

\section{Cancer, Infection, and Immunology: Intersec- tions in Translational and Clinical Science}

Elad Sharon, M.D., M.P.H., joined the NCI Cancer Therapy Evaluation Program (CTEP) in December 2011 as a Senior Investigator in the Investigational Drug Branch, where he works with academia and industry to develop promising new cancer therapies. His portfolio includes antibody-drug conjugates, immune checkpoint inhibitors, and other agents. As part of his work in immunotherapy drug development, he has made a major effort to advance the understanding of immune-related adverse events, including the establishment of the Alliance$\mathrm{NIH}$ immune-related adverse events (irAE) biorepository. In addition, $\mathrm{He}$ is the co-Principal Investigator of the Study of Nivolumab in Patients with Autoimmune Disorders and Advanced Malignancies (AIM-NIVO) trial, evaluating the use of nivolumab for patients with pre-existing autoimmune disease.

He received his MD from Baylor College of Medicine in Houston, Texas, in 2003. He completed his internal medicine residency at Emory University in 2006 and his Hematology/ Oncology Fellowship at NIH in 2011 while obtaining a Master of Public Health degree at Harvard University in 2009. His fellowship research focused on clinical trials in mesothelioma.

His interest in the intersection of autoimmunity and tumor immunology is focused on translational frameworks primarily using samples from patients on clinical trials. These samples can be shared with translational and basic scientists to find new insights into the nexus between autoimmune conditions and immune-related adverse events. The same factors that influence how a cancer patient may respond to a certain immune-based anti-cancer therapy can also influence how an immune-related adverse event may emerge. This involves factors such as host genetics, lifetime environmental exposure, T-cell repertoire, carcinogenesis, tumor evolution, and treatment response.

Many of the types of immunotherapies that have been developed over the past several decades focus on perturbing normal components of immune homeostasis. There are a whole host of other immune checkpoints that are present that affect whether a given $\mathrm{T}$ cell with a specific T-cell receptor (TCR) might be able to recognize a major histocompatibility complex peptide as a target, as well as whether or not the patient is going to be able to ultimately have a response [12].

As mentioned before, the spectrum of anti-PD-1 and PDL1 antagonist activity is enormous. Many drugs have been approved in this class. There are many being developed in the United States as well as other parts of the world. Nivolumab was a joint Japan-United States project involving BMS and Ono Pharmaceutical, focused on this area [13]. Given the breadth of activity, immunotherapy is also associated with immune-related adverse events. The best approach to find the 
mechanism of therapy-related adverse events is to obtain a breadth of patient tissue or blood samples that are usually being "left on the table" during clinical trials. Without the effort to find the cause of these adverse events, patients that have adverse events are not appropriately managed, or properly and fully interrogated, so knowledge is lost on how to address these events in the future.

Methods to obtain answers regarding these problems include: 1) analyzing specific cases through imaging and archives of images (magnetic resonance imaging (MRI)/computed tomography (CT) scans) as well as creating an established, well-annotated biorepository. With these datasets, machine learning can be used to derive new insights; 2) studying specific vulnerable populations; 3 ) analysis of electronic health data records; 4) better categorizing, managing and collecting information from irAEs (immunotherapy agent associated adverse event (AE) terms on common terminology criteria for adverse events (CTCAE), use of management guidelines, test treatment strategies for irAEs in clinical trials); and 5) casecontrol studies from clinical trials (examine HLA, microbiota, or other differences from patients who experience irAEs and those who do not).

The goal of translational studies is to identify at-risk patients using clinical risk factors (e.g., medical history of heart disease) to better understand biomarker stratification, prevent harm or mitigate risks of immunotherapy, and understand the pathobiology of the given adverse event. Some patients who received PD-1 inhibitor treatment developed a previously unknown infection with tuberculosis (TB) [14].

The NCI and the Alliance for Clinical Trials in Oncology elected to develop a biorepository using tissue from patients in clinical trials that receive immune checkpoint inhibitors and experience adverse events, the purpose of which was to establish an efficient and centralized repository for acquisition and organized distribution of well-annotated biospecimens to improve the understanding of molecular pathogenesis of severe irAEs. This includes pre-treatment tissue from patients as well as retrospective samples of patients that experienced adverse events. The irAE Biorepository, once fully accrued, may allow for additional insights to be gathered from adverse events that are currently poorly understood and infrequent, but responsible for significant patient morbidity and mortality.

\section{Mycobacterial Infection and Immunotherapy}

Shunsuke Sakai, Ph.D., is a Staff Scientist at the National Institute of Allergy and Infectious Diseases (NIAID) where he works for the T Lymphocyte Biology Section in the Laboratory of Parasitic Diseases. He received his Master of Science and Ph.D. from Kyoto University. His research focuses on understanding mechanisms of immunity to pulmonary pathogens including Mycobacterium tuberculosis, the causative agent of TB.

TB remains a leading cause of global mortality and morbidity. The first and only vaccine for TB, bacillus CalmetteGuerin (BCG), does not confer lasting protection against pulmonary disease in adults. Given the long duration of anti-TB treatment regimens and the emergence of drug-resistant TB, the development of effective prophylactics and therapeutics is urgently needed.

T-cell immunity is necessary to control TB but is unable to eradicate the bacterial infection. Therefore, strategies to manipulate T-cell effector function may have the potential to achieve better control of TB. Boosting T-cell function by targeting the PD-1 immune checkpoint pathway has been well established for the treatment of different types of cancer. Thus, PD-1 blockade has been suggested as a host-directed therapy for TB. Unexpectedly, he discovered that the loss of PD-1-mediated inhibition exacerbates TB in both mice and non-human primate models $[15,16]$. Furthermore, there is a growing number of case reports in the literature documenting TB associated with PD-1 blockade in cancer patients. He also showed that TB following PD-1 blockade for cancer immunotherapy is associated with increased TB-specific T-cell responses in the circulation [14].

In conclusion, TB is a potential adverse event of immune checkpoint blockade-based cancer therapy. Data from multispecies indicate that PD-1 blockade exacerbates TB infection and thus is unlikely to be useful as a host-directed therapy for TB.

\section{NIH Grants}

William Timmer, Ph.D., works as the Program Director for the CTEP at the NCI. He manages a portfolio of clinical grants involving the brain, head and neck, lung, liver, colorectal, pancreatic, and sarcoma cancers. He is the CTEP representative to the Informatics Technology for Cancer Research program, as well as the Program Director for the Adult Brain Tumor Consortium, the Childhood Cancer Survivor Study, the Neurofibromatosis NF-1 Consortium, and the Drug Resistance and Sensitivity Network.

He received his Ph.D. in Physical Chemistry from the University of Wisconsin-Milwaukee, where he intended to pursue a career in the physical sciences. However, a unique opportunity became available at $\mathrm{NIH}$, and he transitioned to molecular biology and clinical sciences. He studied the immunoregulatory effects of cytokines on HIV replication in the Laboratory of Immunoregulation at the NIAID, where he was mentored by Dr. Anthony Fauci.

He presented the structure of NCI and practical tips for applying for grants. In his role as Program Director (of which there are only three that manage grants with clinical trials), his responsibilities include: 1) advising grantees and grant applicants; 2) attending scientific meetings and keeping track of the latest scientific breakthroughs and publications; 3) reviewing letters of intent (LOIs) that are submitted to CTEP; 4) presenting grant to NCI Associate Director and at senior program leader meetings; 5) monitoring grant progress; and 6) developing new initiatives.

He advised prospective grant applicants to use the NIH Office of Extramural Website to find information about grants and to consult program announcements for ongoing solicitations with specific scope and objectives. Meanwhile, requests 
for applications are issued by NCI as one-time requests with defined research topics, with grants reviewed in a specific research institute.

The budget for a modular R01 is $\$ 25,000$ per module with a maximum of 10 modules per year, thus constituting $\$ 250,000$ per year for a total of 5 years, meaning up to $\$ 1.25$ million is available for direct research costs over 5 years. Non-modular budgets can propose up to $\$ 499.999$ in direct costs per year. For P01 grants, the maximum budget is limited to approximately $\$ 1.8$ million for first-year direct costs. R21 grants are limited to $\$ 275,000$ over 2 years.

His advice on writing a good grant application mainly concerning R01 grants but applying to all grant mechanisms emphasized the "specific aims" page of the grant application. Best practices include: 1 ) presenting a logical flow of information, from general information to the final statement; 2) outlining the significance of the problem clearly; 3 ) discussing how the project will impact the field; 4) a description of preliminary data; and 5) a hypothesis.

He also advised that paragraphs should be no more than three to five sentences to compel the reviewer to keep reading and that they should be written in the active tense to emphasize the performance of the action (e.g., "we have analyzed the results...") to the reader. The structure should include a brief introduction to orient the review, with a final sentence outlining what can be accomplished by the successful completion of the study to keep the reviewer engaged. In addition, graphics and pictures should always be included on the left-hand side of the page, given the left-to-right method of reading in English.

Project program grants ( $\mathrm{P} 01)$ are for senior researchers and can include or not include may include a clinical trial. These are reviewed by NCI and funded based on their impact score. He advised emphasizing the unique and beneficial aspects of the proposal to justify the benefits it would deliver to patients and the research community.

He distinguished between his role as Program Director and the review committees; his role is to serve as a point of contact for principal investigators to answer grant questions; Scientific Review Officers set up study sections to review the grant; peerreview information is then included in a Summary Statement which is available to the investigator that submitted the grant.

William Timmer closed his presentation with a key point that the NCI funds what meets their "pay line" (scoring threshold), and that developing a relationship with the Program Director is very important to secure a grant advisor and grant advocate to maximize the likelihood of success, improve the chance of a grant scoring well.

\section{Concluding Remarks}

The conference proved successful as the number of participants was increased significantly due to it being simultaneously online in Japan. Excellent presentations and active discussions occurred regarding the theme of the meeting on cancer and infectious diseases, an emerging conceptual link in translational oncology research. We examined the relationship from many different angles through the talks by the distinguished presenters. Just as importantly, they shared their thoughts on career development with the many young investigators in the audience. The conference provided a superb opportunity to develop collaborations among academic researchers, investigators, and pharmaceutical companies in both countries.

The event, co-hosted by governmental organizations, was unique in its mission and target audience of academia, pharma, and policymakers who engage in grant funding and drug approval. Moving forward, future conferences will probably continue to have online sessions to reach interested Japan based participants, while restarting in person meetings in the US. Therefore, such conferences may work as an effective tool of science-policy communication and be beneficial in advancing medicine internationally.

\section{Authors (continued)}

${ }^{\mathrm{j} C a n c e r}$ Therapy Evaluation Program, Division of Cancer Treatment and Diagnosis, National Cancer Institute (NCI), National Institutes of Health (NIH), Bethesda, MD 20892, USA

${ }^{\mathrm{k}} \mathrm{T}$ Lymphocyte Biology Section, Laboratory of Parasitic Diseases, National Institute of Allergy and Infectious Diseases (NIAID), National Institutes of Health (NIH), Bethesda, MD 20892, USA

${ }^{1}$ Cancer Therapy Evaluation Program, National Cancer Institute (NCI), National Institutes of Health (NIH), Bethesda, MD, USA

mSystemic Autoimmunity Branch, National Institute of Arthritis and Musculoskeletal and Skin Diseases (NIAMS), National Institutes of Health (NIH), Bethesda, MD 20892, USA

nOral Immunology and Inflammation Section, National Institute of Dental and Craniofacial Research (NIDCR), National Institutes of Health (NIH), Bethesda, MD 20892, USA

oLaboratory of Receptor Biology and Gene Expression, National Cancer Institute, National Institutes of Health, Bethesda, MD 20892, USA

pOffice of Research and Standards, Office of Generic Drugs, Center for Drug Evaluation and Research, US Food and Drug Administration, Silver Spring, MD, USA

${ }^{q}$ Liver Diseases Branch, National Institute of Diabetes and Digestive and Kidney Diseases (NIDDK), National Institutes of Health (NIH), Bethesda, MD 20892, USA

${ }^{\mathrm{E}}$ Embassy of Japan in the USA

sDartmouth-Hitchcock Medical Center, Lebanon, NH 03756, USA

tLaboratory of Systems Cancer Biology, The Rockefeller University, New York, NY 10065, USA

"Breast Surgery, Department of Surgical Oncology, Roswell Park Comprehensive Cancer Center, Buffalo, NY 14263, USA

'Department of Surgery, University at Buffalo Jacobs School of Medicine and Biomedical Sciences, The State University of New York, Buffalo, NY 14263, USA wepartment of Surgery, Niigata University Graduate School of Medical and Dental Sciences, Niigata 951-8510, Japan

xDepartment of Breast Surgery and Oncology, Tokyo Medical University, Tokyo 160-8402, Japan

yepartment of Gastroenterological Surgery, Yokohama City University Graduate School of Medicine, Yokohama 236-0004, Japan

${ }^{2}$ Department of Breast Surgery, Fukushima Medical University School of Medicine, Fukushima 960-1295, Japan

\#Division of Cancer Treatment and Diagnosis, National Cancer Institute, NIH, Bethesda, MD, USA

*Corresponding Authors: Hiroto Nakano, Translational Genetics and Genomics Unit, National Institute of Arthritis and Musculoskeletal and Skin Diseases (NIAMS), National Institutes of Health (NIH), Bethesda, MD 20892, USA. Email: hiroto.nakano@nih.gov; Naoko Takebe, Division of Cancer Treatment and Diagnosis, National Cancer Institute (NCI), National Institutes of Health (NIH), Bethesda, MD 20892, USA. Email: Takeben@mail.nih.gov

doi: https://doi.org/10.14740/wjon1422 


\section{Acknowledgments}

We would like to thank the participants for their comments, questions, and feedbacks. This conference would not have been possible without the generous support of our patients and our main sponsor: Embassy of Japan in the USA (Washington, D.C., USA). We appreciate the kind support by the Japan Agency for Medical Research and Development (AMED) Washington D.C. Office.

\section{Financial Disclosure}

The work was supported in part by a Grant for global education and research center aiming at the control of AIDS (Global Center of Excellence supported by Monbu-Kagakusho) (H.M.), in part by a grant for Research on HIV/AIDS from the Ministry of Health, Welfare, and Labor of Japan (H.M.), in part by a grant for Development of Novel Drugs for Treating COVID-19 from the Intramural Research Program of National Center for Global Health and Medicine (H.M., 19A3001), in part by Japan Agency for Medical Research and Development (AMED) (H.M., 20fk0108257, 20fk0108260, 20fk0108510, 20fk0108502) and by the Intramural Research Program of Center for Cancer Research, National Cancer Institute, National Institutes of Health (H.M.).

\section{Conflict of Interest}

Y.Y.J. has received research funding provided to her institution from Bayer, Bristol-Myers Squibb, Cycle for Survival, Department of Defense, Eli Lilly, Fred's Team, Genentech/Roche, Merck, NCI, and RGENIX. She has performed advisory boards/ consulting activities for Astra Zeneca, Basilea Pharmaceutica, Bayer, Bristol-Myers Squibb, Daiichi-Sankyo, Eli Lilly, Imugene, Merck, Merck Serono, Michael J. Hennessy Associates, Paradigm Medical Communications, Pfizer, RGENIX, Seagen, and Zymeworks Inc. She has stock option interest with RGENIX. All other authors declare no competing interests.

\section{Author Contributions}

Conceptualization: H.N., T.M., and N.T. Writing - original draft preparation: H.N., T.M., Y.Y.J., S.M., H.M., N.T.U., E.S., S.S., and W.C.T. Writing - review and editing: N.T. and T.I. Supervision: S.N., S.F., M.K., T.I., H.K., and K.S. Project administration: S.N., T.Ik., T.In., T.M., H.K., N.Y., K.T., and N.T. All authors have read and agreed to the published version of the manuscript.

\section{Data Availability}

The data supporting the findings of this study are available from the corresponding author upon reasonable request.

\section{Disclaimer}

This article reflects the views of the author and should not be construed to represent FDA's view or policies.

\section{References}

1. Seo S, Ryu MH, Park YS, Ahn JY, Park Y, Park SR, Ryoo BY, et al. Loss of HER2 positivity after anti-HER2 chemotherapy in HER2-positive gastric cancer patients: results of the GASTric cancer HER2 reassessment study 3 (GASTHER3). Gastric Cancer. 2019;22(3):527-535.

2. Chung HC, Bang YJ, C SF, Qin SK, Satoh T, Shitara $\mathrm{K}$, Tabernero J, et al. First-line pembrolizumab/placebo plus trastuzumab and chemotherapy in HER2-positive advanced gastric cancer: KEYNOTE-811. Future Oncol. 2021;17(5):491-501.

3. Mine S, Hishima T, Suganuma A, Fukumoto H, Sato Y, Kataoka M, Sekizuka T, et al. Interleukin-6-dependent growth in a newly established plasmablastic lymphoma cell line and its therapeutic targets. Sci Rep. 2017;7(1):10188.

4. Rous P. A Transmissible avian neoplasm. (Sarcoma of the Common Fowl.). J Exp Med. 1910;12(5):696-705.

5. Epstein MA, Achong BG, Barr YM. Virus particles in cultured lymphoblasts from Burkitt's lymphoma. Lancet. 1964;1(7335):702-703.

6. Katano H, Hishima T, Mochizuki M, Kodama Y, Oyaizu N, Ota Y, Mine S, et al. The prevalence of opportunistic infections and malignancies in autopsied patients with human immunodeficiency virus infection in Japan. BMC Infect Dis. 2014;14:229.

7. Mitsuya H. Fight against COVID-19 but avoid disruption of services for other communicable diseases (CDs) and noncommunicable diseases (NCDs). Glob Health Med. 2020;2(6):343-345.

8. Maeda K, Higashi-Kuwata N, Kinoshita N, Kutsuna S, Tsuchiya K, Hattori SI, Matsuda K, et al. Neutralization of SARS-CoV-2 with IgG from COVID-19-convalescent plasma. Sci Rep. 2021;11(1):5563.

9. Takamatsu Y, Imai M, Maeda K, Nakajima N, HigashiKuwata N, Iwatsuki-Horimoto K, Ito M, et al. Highly neutralizing COVID-19 convalescent plasmas potently block SARS-CoV-2 replication and pneumonia in syrian hamsters. J Virol. 2021:JVI0155121.

10. Bafna K, Krug RM, Montelione GT. Structural similarity of SARS-CoV2 M(pro) and HCV NS3/4A proteases suggests new approaches for identifying existing drugs useful as COVID-19 therapeutics. ChemRxiv. 2020.

11. Hattori SI, Higashi-Kuwata N, Hayashi H, Allu SR, Raghavaiah J, Bulut H, Das D, et al. A small molecule compound with an indole moiety inhibits the main protease of SARS-CoV-2 and blocks virus replication. Nat Commun. 2021;12(1):668.

12. Murciano-Goroff YR, Warner AB, Wolchok JD. The future of cancer immunotherapy: microenvironment-targeting combinations. Cell Res. 2020;30(6):507-519.

13. Paz-Ares LG, Ramalingam SS, Ciuleanu TE, Lee JS, Ur- 
ban L, Caro RB, Park K, et al. First-line nivolumab plus ipilimumab in advanced NSCLC: 4-year outcomes from the randomized, open-label, Phase 3 CheckMate 227 Part 1 Trial. J Thorac Oncol. 2021.

14. Barber DL, Sakai S, Kudchadkar RR, Fling SP, Day TA, Vergara JA, Ashkin D, et al. Tuberculosis following PD-1 blockade for cancer immunotherapy. Sci Transl Med. 2019;11(475).

15. Kauffman KD, Sakai S, Lora NE, Namasivayam S, Baker
PJ, Kamenyeva O, Foreman TW, et al. PD-1 blockade exacerbates Mycobacterium tuberculosis infection in rhesus macaques. Sci Immunol. 2021;6(55).

16. Sakai S, Kauffman KD, Sallin MA, Sharpe AH, Young HA, Ganusov VV, Barber DL. CD4 T cell-derived IFNgamma plays a minimal role in control of pulmonary mycobacterium tuberculosis infection and must be actively repressed by PD-1 to prevent lethal disease. PLoS Pathog. 2016;12(5):e1005667. 